\title{
Gorgona, Baudó y Darién (Chocó Biogeográfico, Colombia): ecorregiones modelo para los estudios ecológicos de comunidades de quebradas costeras
}

\author{
Juan F. Blanco ${ }^{1 *}$, Camilo Escobar-Sierra ${ }^{1} \&$ Juan D. Carvajal-Quintero ${ }^{1}$ \\ 1. Instituto de Biología, Universidad de Antioquia. A. A. 1226. Medellín (Antioquia), Colombia; \\ blanco@exactas.udea.edu.co; miloes114@gmail.com; juanchocarvajal@gmail.com
}

\author{
Recibido 18-X-2013. Corregido 20-XI-2013. Aceptado 19-XII-2013.
}

\begin{abstract}
Gorgona, Baudó and Darién (Biogeographic Chocó, Colombia): model ecoregions for coastal stream ecology studies. This paper summarizes the available information on the geology, hydrology, and biota of coastal streams located in the Gorgona National Natural Park (GNNP), and the Baudó and Darién ecoregions within the Biogeographic Chocó (Colombia). Despite of the scant number of studies, we hypothesized that these streams are consistent with the typology observed in volcanic oceanic islands in the Caribbean and the Pacific that do not conform to the tenets of the River Continuum Concept. Coastal streams in the Biogeographic Chocó are short $\left(10^{0}-10^{1} \mathrm{~km}\right)$ and steep and waterfalls and cascades are frequent due to tectonic origin. Stepand-pool sequences are common in montane reaches, while cobble-bed glides and riffles dominate in coastal plain reaches. Flow regimes are flashy in Pacific drainages (annual rainfall: $<7000 \mathrm{~mm}$ ), but seasonally dry in the Caribbean drainages $(<3500 \mathrm{~mm})$. Therefore, floods and droughts are important drivers of communities and ecosystem processes. Canopies are closed in low-order streams discharging directly to the ocean, thus contributing large amounts of litter. Diadromous fish and shrimp dominate consumer assemblages and various species are shared with streams in the Central America and the Antilles. These species play ecological roles probably equivalent to those found in other regions. These coastal streams are unique in terms of the number of primary freshwater fishes found (some endemics), and the functionally absence of diadromous gastropods. Rev. Biol. Trop. 62 (Suppl. 1): 43-64. Epub 2014 February 01.
\end{abstract}

Key words: coastal streams, neotropics, diadromous fauna, aquatic insects, stream ecology, river continuum concept.

Las investigaciones realizadas en quebradas insulares y costeras neotropicales han contribuido significativamente al desarrollo de la ecología lótica. Sin embargo, debido a las diferencias geográficas dentro del Trópico, y entre éste y las zonas templadas, es necesario establecer qué tan generales son los patrones comunitarios y ecosistémicos allí encontrados (Boulton et al., 2008; Jacobsen, Cressa \& Dudgeon, 2008; Boyero, Ramírez, Dudgeon \& Pearson, 2009). Actualmente se acepta que los sistemas lóticos más estudiados del Neotrópico (Costa Rica y Puerto Rico: Ramírez \& Hernández-Cruz, 2004; Blanco \& Scatena, 2006; Greathouse \& Pringle, 2006; Ramírez,
Pringle \& Douglas, 2006) son similares a los del océano Pacifico (e.g. Hong Kong, Hawaii, Polinesia Francesa y Nueva Zelanda; Winterbourn, Rounick \& Cowie, 1981; Resh \& De Szalay, 1995; Dudgeon, 2000; Craig 2003; Smith, Covich \& Brasher, 2003) en términos de la composición taxonómica y las historias de vida de la fauna acuática (baja abundancia de insectos, ausencia de ordenes con poca capacidad de migración y sensibilidad fisiológica [Plecoptera, Ephemeroptera, Megaloptera], y dominancia en biomasa de moluscos, crustáceos y peces diádromos). Por lo tanto, las hipótesis generadas en estos sistemas del Caribe tienen una relevancia de carácter global. Por el 
contrario, las quebradas de las islas y costas del Pacífico Oriental Tropical (POT), y particularmente las de la Provincia del Chocó Biogeográfico que se expande entre Ecuador y Colombia, han sido poco estudiadas. Los estudios e inventarios disponibles para las costas e islas del POT se concentran en los parques nacionales (e.g. Corcovado-Costa Rica, Coiba-Panamá, Gorgona-Colombia; Lyons \& Scheneider, 1990; Scheneider \& Lyons, 1993; Boyero \& Bailey, 2001; Gómez-Aguirre, Longo-Sánchez \& Blanco, 2009), y usualmente son producto de expediciones científicas de corta duración.

El Parque Nacional Natural Gorgona (PPNG), ubicado a 35 kilómetros de la costa en el sur del Pacífico colombiano (denominado Isla Ciencia por la gran cantidad de investigaciones científicas que se han desarrollado en ella), es un ejemplo del rezago de la investigación en los sistemas lóticos en comparación a otras disciplinas. Desde su declaración como área natural protegida en 1985, solamente se realizaron dos inventarios de los macroinvertebrados acuáticos, y solo en 2008 se realizó el primer estudio ecológico con el objetivo de comprender cuantitativamente los patrones de distribución espacial de los macroinvertebrados acuáticos y sus causas y describir la historia natural del entorno (Blanco, Ramírez \& Scatena, 2009; Gómez-Aguirre et al., 2009; Gómez-Aguirre 2009; Longo-Sánchez \& Blanco 2014a; 2014b en este número).

Los primeros resultados apoyaron la idea de que las comunidades de macroinvertebrados acuáticos del PNNG presentaban características similares a los ensamblajes de otras islas oceánicas y costeras del Pacífico y del Caribe, tales como la baja densidad de insectos, la dominancia en número y en biomasa de pocos grupos de insectos, la ocurrencia de muchos grupos poco frecuentes o raros y la dominancia en términos de biomasa de crustáceos diádromos (Gómez-Aguirre, 2009; Gómez-Aguirre et al., 2009). Aunque ningún orden estuvo ausente por la cercanía al continente, la entomofauna mostró rasgos de insularidad como la presencia de una o pocas morfoespecies por familia y la mayor riqueza de Trichoptera, Coleoptera y Diptera (Gómez-Aguirre, 2009). Adicionalmente, otras características como los cursos cortos y los regímenes de caudal torrenciales (Blanco, 2009a; 2009b) las hicieron homogéneas en términos faunísticos y de algunas propiedades físico-químicas (Blanco, 2009b; Gómez-Aguirre et al., 2009), desviándolas de las predicciones del mayor paradigma de organización longitudinal de los ecosistemas lóticos, el Concepto del Río Continuo (CRC, Vannote, et al., 1980), el cual tampoco se ha ajustado a otros sistemas loticos insulares de zonas tropicales y templadas (Winterbourn et al.,1981; Greathouse \& Pringle, 2006; Turner, Williams \& Alkins-Koo, 2008; Hyslop \& Hunte-Brown, 2012).

Por lo anterior, propusimos que las quebradas del PNNG podrían ser sitios de referencia (con baja intervención humana) que representan la generalidad ecológica de las quebradas costeras del POT y que además podían ser utilizadas como modelos de estudio para someter a prueba hipótesis generadas en sistemas lóticos insulares y costeros tropicales y subtropicales (Blanco et al., 2009). Este artículo aporta datos cuantitativos y cualitativos obtenidos en el campo y la literatura de quebradas de las ecorregiones o distritos Baudó y Darién dentro del Chocó Biogeográfico colombiano, juntamente con los datos de nuestras investigaciones en PNNG, para: (1) establecer la generalidad de los patrones de ensamblaje comunitario observados en el PNNG; (2) proponer una tipología de las quebradas costeras del Chocó Biogeográfico por medio del planteamiento de hipótesis que puedan ser sometidas a prueba; y (3) realizar comparaciones con las quebradas insulares de otras regiones del mundo. Para la comparación se utilizó información sobre la geología y geomorfología, la hidroclimatología y las comunidades acuáticas.

\section{MATERIALES Y MÉTODOS}

Se recopiló y analizó la bibliografía publicada hasta 2012 sobre las quebradas costeras del PNNG (ecorregión Gorgona), y las ecorregiones Baudó (Pacífico Norte colombiano) 
y Darién (Caribe Sur colombiano). Se revisaron bases de datos en línea y se visitaron diferentes bibliotecas. Se incluyeron libros, artículos científicos, tesis e informes técnicos. Adicionalmente, se utilizó información de campo obtenida por los autores durante 20082009 en PNNG, 2009 en el PNN Utría (ecorregión Baudó), y 2011 en Capurganá (ecorregión Darién) (Cuadro 1). La información obtenida fue analizada y resumida por sitio, presentando las características geológicas e hidrológicas, y un análisis de la fauna lótica encontrada. Asimismo, se elaboró un mapa donde se mostraron las tres ecorregiones y las localidades estudiadas y sus principales quebradas costeras. Las localidades estudiadas en este trabajo se ubican en el Chocó Biogeográfico, que se extiende desde la provincia panameña de Darién y el golfo de Urabá en Colombia, en el norte, hasta la provincia ecuatoriana de Manabí, en el sur; y entre el océano Pacífico y la divisoria de aguas de la vertiente occidental de la cordillera de Los Andes, que se eleva desde el nivel del mar hasta aproximadamente $3500 \mathrm{msnm}$. Esta región es uno de los puntos calientes de biodiversidad terrestre y acuática, y prioridad de conservación (Myers, Mittermeier, Mittermeier, da Fonseca \& Kent, 2000), pero al mismo tiempo altamente amenazada por el rápido crecimiento poblacional (Cincotta, Wisnewski \& Engelman, 2000). Recientemente, Abell et al. (2008) renombró a la región como "Vertiente Pacífica de los Andes del Norte y Cuenca del río Atrato" en una clasificación ecorregional mundial basada en peces dulceacuícolas.

Geología e hidrología del Chocó Biogeográfico: El origen de las quebradas costeras de esta subprovincia biogeográfica está asociado con la convergencia de las placas de Nazca, Suramericana y Caribe durante el Terciario, la cual dio origen al levantamiento orogénico que forma las actuales serranías costeras de San Blas-Darién y de Majé-Baudó (Page, 1986; Galvis \& Mojica, 1995). Este proceso llevó a que hace tan solo tres millones de años, durante el Plioceno se formara completamente el istmo de Panamá y se diera la separación definitiva del mar Caribe y el océano Pacífico, generando un sistema de cuencas hidrográficas costeras relativamente cortas en ambas vertientes (Martínez, 1995; IGAC-INGEOMINAS, 2005), dominadas por una litología ígnea y metamórfica que no permite un desarrollo profundo de los suelos (Galvis \& Mojica, 1995; Lombana, 1995). Estos relieves costeros de varios cientos de metros de altura modifican los movimientos del aire y las corrientes de convección ascendentes, generando sistemas de circulación mar-costa, valle-montaña, lluvia orogénica y sombra de lluvia. Sin embargo, al sur de Cabo Corrientes en el Pacífico colombiano el relieve costero desaparece, presentándose una amplia planicie que colinda con las estribaciones de la cordillera Occidental, varias decenas o cientos de kilómetros tierra adentro, formando grandes ríos (Lobo-Guerrero, 1995; Martínez 1995).

CUADRO 1

Resumen de los métodos de muestreo usados por los autores para obtener la información primaria.

TABLE 1

Summary of the sample methods used by the authors to obtain the primary data.

\begin{tabular}{|c|c|c|}
\hline & Darién & Gorgona \\
\hline & \multicolumn{2}{|c|}{ Métodos de muestreo } \\
\hline Número de cursos & 3 & 9 \\
\hline Método de colecta & Red Surber $\left(0,09 \mathrm{~m}^{2}, 0,5 \mathrm{~mm}\right.$ ojo de malla). & Red Surber $\left(0,09 \mathrm{~m}^{2}, 0,5 \mathrm{~mm}\right.$ ojo de malla). \\
\hline Metodología & $\begin{array}{l}\text { Muestreo hábitat múltiple (Alba-Tercedor, } \\
\text { Pardo, Prat \& Pujante, 2005). }\end{array}$ & $\begin{array}{l}\text { "Jerarquía subyacente" (Parsons, Thoms \& } \\
\text { Norris, 2004). }\end{array}$ \\
\hline Época muestreo & Junio-Julio de 2011. & Abril y Junio 2008. \\
\hline Referencia estudio & Contreras, 2012. & Gómez-Aguirre et al., 2009. \\
\hline
\end{tabular}


La influencia de la Zona de Convergencia Intertropical (ZCIT), hace que cuencas hidrográficas costeras del Chocó Biogeográfico norte tengan uno de los climas más húmedos del mundo (revisado por Blanco, 2009a). De hecho se afirma que el Chocó Biogeográfico cuenta con quizás el más vasto complejo fluvial del mundo (Díaz-Merlano \& Gast-Harders, 2009), ya que el promedio anual de lluvias sobrepasa los $10000 \mathrm{~mm}$, y los valores mensuales máximos alcanzan los $3000 \mathrm{~mm}$. La alta temperatura (rango: $27-30^{\circ} \mathrm{C}$ ) genera altos valores de humedad relativa $(<88 \%)$ y evapotranspiración que refuerzan los niveles de precipitación a través de procesos locales. La precipitación media muestra un régimen bimodal, donde la primera temporada de lluvia (abril-junio) es más débil que la segunda (setiembre-noviembre) (Eslava, 1995; Blanco, 2009a).

Debido a la posición costera de las serranías del Baudó, del Darién y otras menores del Chocó Biogeográfico norte, que genera cuencas hidrográficas pequeñas, y a la gran cantidad de precipitación que se recibe estacionalmente o episódicamente, la región posee una gran cantidad de ríos, quebradas y arroyos de cursos cortos y ordenes bajos con regímenes (hidrógrafas) torrenciales, aunque la mayoría de ellos no se encuentran aforados o instrumentados (Lobo-Guerrero, 1995; Blanco, 2009a; 2009b). Estos cursos drenan valles aluviales de pendientes pronunciadas, que se caracterizan por estar encajados entre dos salientes y se configuran como zonas deprimidas cubiertas por depósitos sedimentarios cuaternarios (Díaz-Merlano \& Gast-Harders, 2009; Prüssman, 2012). A pesar de que estos valles están cubiertos por densos bosques lluviosos y húmedos tropicales, los aerosoles marinos influyen fuertemente sobre las características fisicoquímicas de las corrientes de agua (e.g. PNNG: Blanco, 2009b).

\section{Isla Gorgona (Pacífico colombiano sur)}

Ubicación: La isla Gorgona está ubicada a $35 \mathrm{~km}$ de Punta Reyes al occidente del municipio de Guapi (departamento del Cauca), ubicado en la parte sur de la costa Pacífica colombiana (Fig. 1). La isla Gorgona tiene una longitud de $8,5 \mathrm{~km}$ y un ancho de $2 \mathrm{~km}$. El Parque Nacional Natural Gorgona (PNNG) fue creado en diciembre de 1985, después de su funcionamiento como isla penitenciaria desde 1957 (Blanco et al., 2009; Valencia, Pérez, Lizarazo \& Blanco, 2009). Tiene una extensión total de 61687 ha que incluye a la isla Gorgonilla y otros islotes, y una significativa porción marina (UAESPNN-DTSO, 2005).

Geología y geomorfología: La isla Gorgona es un macizo volcánico continental (Kerr, 2005), con una altura máxima de 330m.s.n.m. El $80 \%$ de la superficie está constituida por rocas ígneas en una serie estratigráfica que comprende peridotitas basales, sobre las cuales se hallan los gabros que están cubiertos por lavas basálticas con flujos de komatíta (revisado por Blanco, 2009b). Por encima de estos flujos se presenta toba ultramáfica $\mathrm{y}$, a continuación, las rocas sedimentarias del terciario y los depósitos cuaternarios, evidentes especialmente en las formaciones de playa y los deslizamientos en los estrechos valles de las numerosas quebradas.

Hidrología: El clima de la Isla es superhúmedo tropical marítimo debido a que la temperatura del aire y la humedad son altas $\left(26^{\circ} \mathrm{C}\right.$ y $90 \%$, respectivamente) durante todo el año. La distribución de la lluvia a lo largo del año es monomodal y totaliza $6661 \mathrm{~mm} / \mathrm{año}$, mientras que la evaporación totaliza solamente $900 \mathrm{~mm} /$ año (Blanco, 2009a). La menor precipitación se produce entre diciembre y abril, mientras que la mayor se presenta entre mayo y noviembre. Este balance hídrico positivo mantiene más de 100 quebradas, 25 de las cuales son permanentes. Estos sistemas drenan por laderas con pendientes fuertes $(50-75 \%)$ y desembocan al mar siendo aun de primer a tercer orden en una planicie aluvial corta. Las cabeceras de fuerte pendiente se caracterizan por tener sustratos dominados por bloques y roca madre mientras que la planicie aluvial es caracterizada por grava y cantos rodados (Fig. 2a). Las 


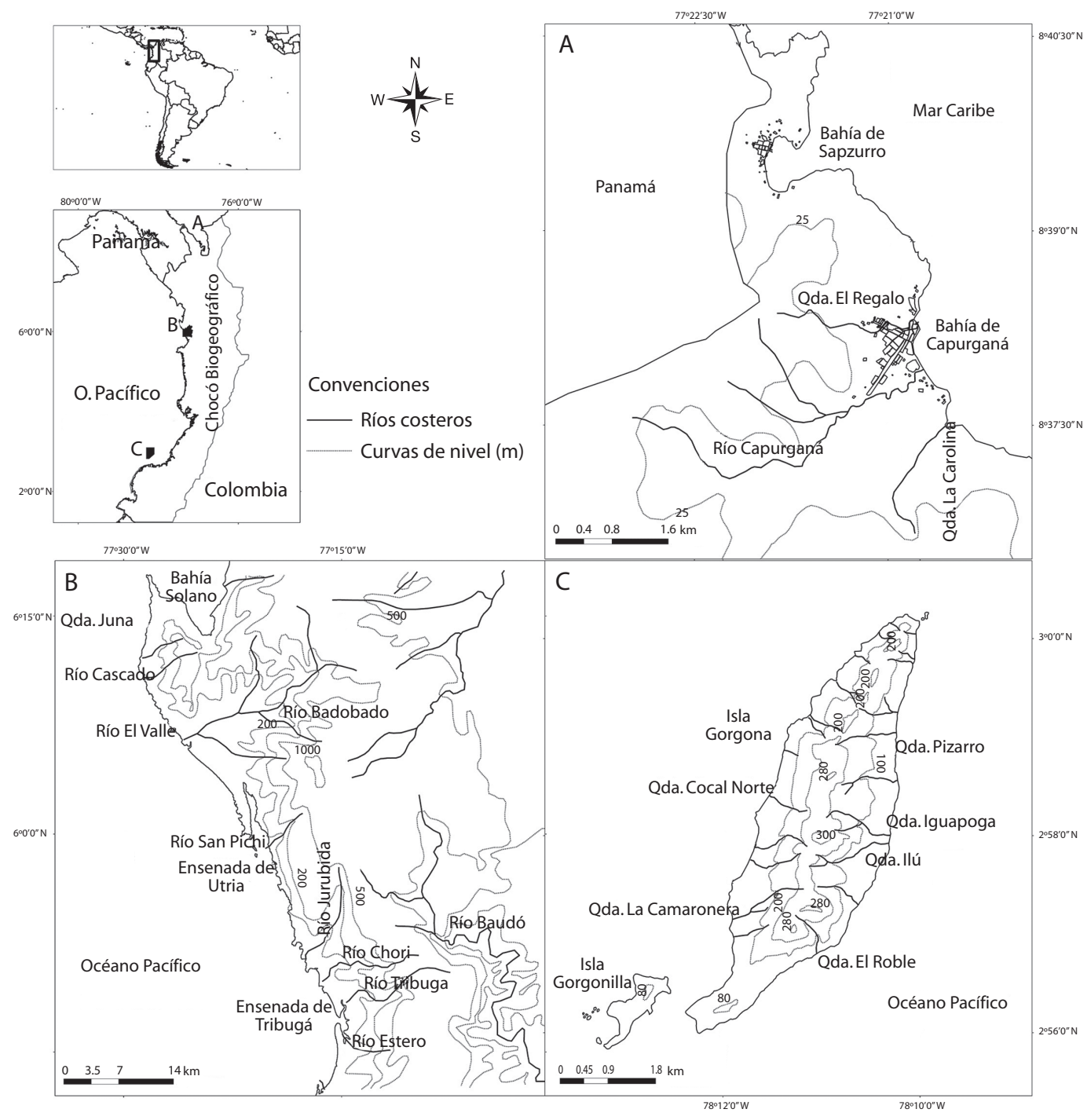

Fig. 1. Ubicación de las localidades estudiadas dentro del Chocó Biogeográfico (Colombia): A) bahía Capurganá y alrededores (ecorregión Darién), B) PNN Utría y alrededores (ecorregión Baudó), C) PNN Gorgona (ecorregión Gorgona). Fig. 1. Location of study areas in the Biogeographic Chocó (Colombia): A) Capurganá Bay and surroundings (Darién Ecoregion), B) Utría NNP and surroundings (Baudó Ecoregion), C) Gorgona NNP (Gorgona Ecoregion).

cinco quebradas más grandes poseen caudales que varían entre 30 y $3001 / \mathrm{s}$, y su variabilidad temporal es controlada por la Zona de Convergencia Intertropical y por la Oscilación Sureña (Blanco, 2009a). En la Isla se encuentra la única quebrada de bajo orden instrumentada en todo el Pacífico colombiano (quebrada Ilú; Blanco, 2009a). Las quebradas son cortas, estrechas y someras, caracterizadas por secuencias de rápidos y piscinas en la planicie costera, y por piscinas y cascadas en la parte montañosa o de alta pendiente; los substratos son predominantemente cantos rodados y bloques, aunque las gravas y cascajos dominan en algunas quebradas con litología sedimentaria (e.g. El Roble y Playa Verde; Gómez-Aguirre et al., 

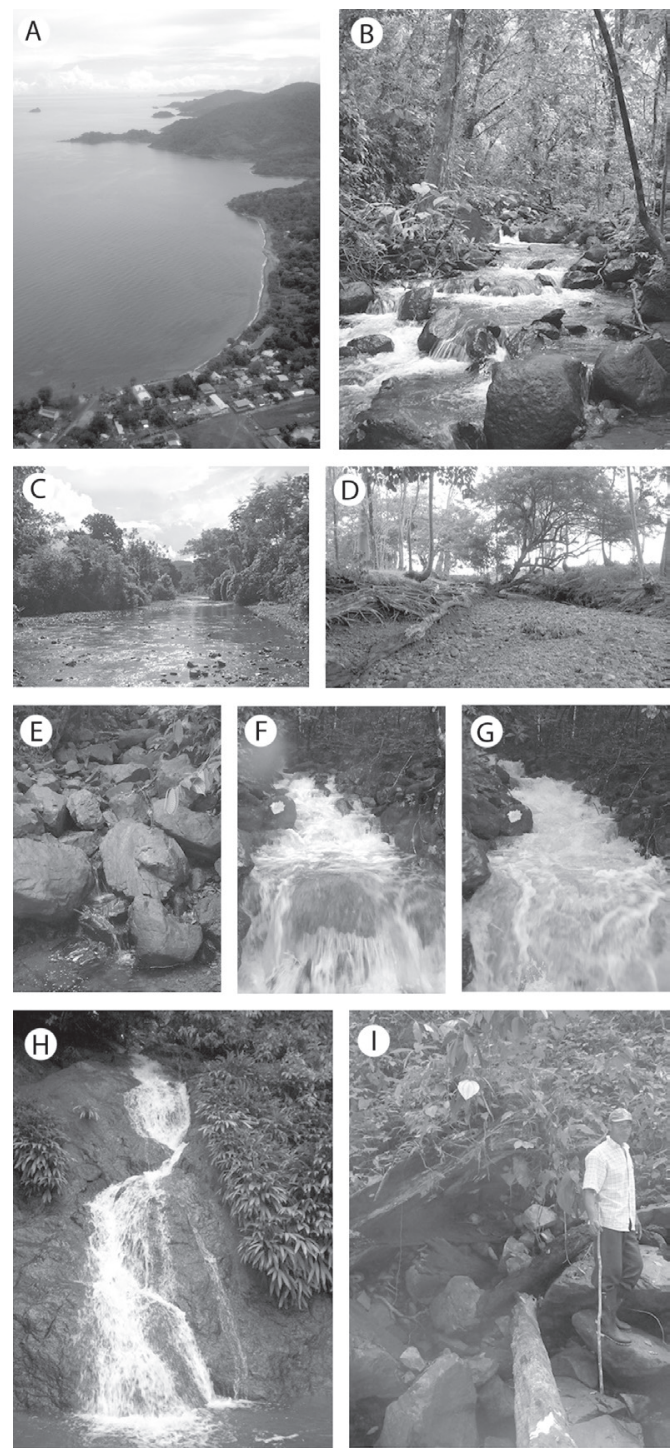

Fig. 2. Características de las quebradas costeras del Chocó Biogeográfico (Colombia): A) Serranías costeras en bahía Capurganá y alrededores (ecorregión Darién); vista hacia el sur. B) Secuencia de piscinas y torrentes en un tramo de la parte media de la quebrada Ilú, PNN Gorgona. C) Tramo de la planicie costera de el río Capurganá (ecorregión Darién), mostrando el lecho de cantos rodados y dosel abierto. D) Cauce seco de la quebrada La Carolina (ecorregión Darién). E, F y G) Secuencia de crecida repentina de la quebrada El Roble, PNN Gorgona durante la época de lluvias. H) Pequeña cascada típica de las quebradas costeras (tributario del río El Valle, cerca a PNN Utría, ecorregión Baudó). I) Árbol caído y movimiento masal de bloques basálticos en una cabecera (quebrada El Roble, PNN Gorgona).
2009; Longo-Sánchez \& Blanco, 2014a en este número). La zona de vida corresponde al Bosque Muy Húmedo Tropical (bmhT), el cual fue intervenido durante el siglo 20 por actividades agropecuarias y penitenciarias. Información detallada y una revisión de la literatura sobre las quebradas del PNNG fue publicada en un número especial por Blanco et al. (2009).

\section{Ensenada de Utría (Serranía del Baudó)}

Ubicación: Esta ensenada es el accidente geográfico más sobresaliente del Parque Nacional Natural Utría (PNNU), ubicado en la costa norte del Pacífico colombiano, en la jurisdicción de los municipios de Bahía Solano, Nuquí, Alto Baudó y Bojayá, del departamento del Chocó (Fig. 1). El PNNU se encuentra en la parte norte del golfo de Tribugá, sobre el flanco occidental de la serranía del Baudó, el cual representa la tipología de la geomorfología, geología y zonas de vida dominantes al norte de cabo Corrientes, el cual marca la divisoria biogeográfica costera entre el Pacífico colombiano norte y sur. El parque fue creado en 1987, y tiene una extensión total (marina y terrestre) de 54300ha (UAESPNN-DTNO, 2006).

Geología y geomorfología: Su origen está relacionado con el levantamiento tectónico de la serranía del Baudó y corresponde a una

Fig. 2. Characteristics of the coastal streams in the Biogeographic Chocó (Colombia). A) Coastal relief in Capurganá Bay and surroundings (Darién Ecoregion), pointing to the South. B) Step and pools sequence in a mid elevation reach in Ilú stream, Gorgona NNP. C) Coastal plain reach in Capurganá River depicting a cobbledominated riverbed and the open canopy. D) Dewatered reach in the coastal plain of La Carolina stream (Darién Ecoregion). E, F y G) Flash-flood sequence in El Roble stream, Gorgona NNP, during the rainy season. H) Low cascade typical of coastal streams (headwater tributary in El Valle River, in the vicinity of Utría NNP, Baudó Ecoregion). I)Large uprooted tree and mass waisting of basalt boulders in a headwater system (El Roble stream, Gorgona NNP). 
megabrecha externa de subducción, formada a inicios del terciario por el rompimiento de la corteza y la subducción del Bloque Oriental. Geológicamente la región se caracteriza por formaciones volcánicas recientes y de sedimentos marinos no consolidados, con porciones de corteza oceánica elevados tectónicamente, razón por la cual su geomorfología es heterogénea, encontrándose largos segmentos de la costa dominados por acantilados basálticos y playas cortas encajadas estrechas en las partes en las que el relieve costero presenta fracturas. La Ensenada se caracteriza por tener rocas basálticas como diabasas, gabros y labradoritas (Galvis, 1980; Galvis y Mojica, 1995).

Hidrología: La estrecha franja costera del PNNU presenta una zona de vida correspondiente al Bosque Muy Húmedo Tropical (bmhT), con una temperatura media superior a $24^{\circ} \mathrm{C}$ y precipitaciones anuales que oscilan entre 4000 y $7000 \mathrm{~mm}$. Esta alta precipitación y relieve costero promueven la formación de un gran número de quebradas de bajo orden, algunas formadas a algunas decenas o cientos de metros de la costa, muchas de ellas desembocando en forma de cascadas o de cursos de alta pendiente (Lobo-Guerrero, 1995). Dentro de la Ensenada sobresalen las quebradas permanentes de ordenes 1-2: La Chunga, La Lemus, Estero Grande, La Aguada y una quebrada sin nombre que cruza el camino a El Valle sobre la península de Utría. Las quebradas y ríos más grandes nacen en la divisoria de la serranía del Baudó, decenas de kilómetros tierra adentro, ya que la parte alta recibe hasta $8000 \mathrm{~mm}$ de precipitación anual (Díaz-Merlano \& Gast-Harders, 2009). Son ejemplo de estos sistemas el río Valle que nace en el Alto El Buey a 1 140m.s.n.m., el río Jurubidá y el río San Pichí. Las quebradas y arroyos que drenan este relieve, generan intensos procesos erosivos que dejan expuesto la roca madre, evitan el desarrollo del suelo, y aportan grandes bloques en los cauces de tramos altos y forman cortas planicies inundables en el litoral, de manera equivalente a lo observado en PNNG (Fig. 2b). Infortunadamente, la información sobre la hidrografía de la región se limita a la publicada dentro de compendios geográficos del Instituto Geográfico Colombiano "Agustín Codazzi" (IGAC, 2006) y hay pocos inventarios y estudios ecológicos (e.g. Fowler, 1944), aunque recientemente, Carvajal-Quintero (2011) realizó un inventario de peces y documentó eventos de migración río arriba.

\section{Bahía de Capurganá (Serranía del Darién)}

Ubicación: La bahía Capurganá se encuentra en el municipio de Acandí, departamento del Chocó, cerca de la frontera Caribe con la República de Panamá (Fig. 1). También en la zona está asentado el territorio colectivo de una comunidad afrodescendiente (Consejo Comunitario Negro del río Acandí y el Chocó Norte) que tiene objetivos de desarrollo sostenible dentro de su plan de etnodesarrollo (E. Pertúz, com. pers). Esta bahía y las vecinas (e.g. Sapzurro) hacen parte de un sistema acantilados basálticos y playas de bolsillo formadas por estribaciones de la serranía de Darién, la cual se extiende desde la desembocadura norte del río Atrato en el golfo de Urabá. La ecorregión Darién es reconocida por su selva húmeda tropical de gran valor natural (Olson \& Dinerstein, 2002), por lo cual presenta varias reservas de la sociedad civil (privadas) y hay varias propuestas para el establecimiento de un parque nacional natural.

Geología y geomorfología: Los relieves de la serranía del Darién son la continuación de la vertiente occidental de la cordillera Occidental y están conformados principalmente por intercalaciones de rocas intrusivas, volcánicas y sedimentarias formadas durante el Cretácico y el Terciario (Prüssman, 2012). Capurganá se encuentra ubicada en un subsistema topográfico paralelo a la costa de relieve escarpado con alturas máximas de 300ms.n.m. y pendientes entre los 30 y los 60\% (Prüssman, 2012). La parte intermareal de algunos sectores está dominada por plataformas arrecifales emergidas (karsos) sobre los que se forman pequeñas 
lagunas costeras y en los que desembocan las quebradas permanentes y efímeras.

Hidrología: El clima de la zona es marítimo influenciado por procesos atmosféricos del mar Caribe y estacionalmente seco (GarcíaValencia, 2007). La temperatura media en la zona es de $27^{\circ} \mathrm{C}$ y la precipitación anual varía entre 2500 y $3000 \mathrm{~mm}$. La distribución de la precipitación es monomodal con un periodo de lluvias entre mayo y noviembre $(<250 \mathrm{~mm} /$ mes) y un periodo seco entre diciembre y abril $(<100 \mathrm{~mm} / \mathrm{mes})$. Esto se refleja en el régimen de caudal marcadamente estacional de los ríos que discurren en la zona. Muchos se secan totalmente en sus tramos bajos y pierden su conectividad con el mar en la época seca y presentan un comportamiento torrencial en la época lluviosa (C. Escobar-Sierra \& J. F. Blanco, observ. pers.). Las quebradas que drenan la zona tienen un área de drenaje pequeña y cursos cortos, desembocando al océano siendo aun de orden bajo o medio. Entre estas sobre salen las quebradas Sapzurro, El Regalo, La Carolina, El Aguacate y el río Capurganá, los cuales nacen a unos cientos de metros o pocos kilómetros de la costa en relieves bajos ( $<100$ m.s.n.m.). Los ríos más grandes son el Acandí y Tolo que nacen aproximadamente a 300m.s.n.m. en un subsistema topográfico del Darién de relieve escarpado y paralelo a la costa (serranía de Tripogadí), y desembocan al mar a través de una fractura (Prüssman, 2012). En general, al igual que las quebradas costeras de la vertiente del Pacífico, tienen una zona de alta pendiente y otra de pendiente suave en la planicie costera. Los sustratos de las zonas de cabecera se caracterizan por estar dominadas por roca madre y bloques basálticos mientras que los valles aluviales se caracterizan por estar encajados entre dos salientes y se configuran como zonas deprimidas cubiertas por depósitos sedimentarios dominados por arenas, gravas y cantos rodados (Prüssman, 2012) (Fig. 2). Al igual que para el Pacífico colombiano norte, la información sobre la hidrografía de la ecorregión Darién se limita a la publicada dentro de compendios geográficos (IGAC, 2006,
García-Valencia, 2007) y hay pocos inventarios y estudios ecológicos (Álvarez-León, 1991; Camargo \& Rozo, 2003; Mena-Moreno, 2010; Contreras, 2012).

\section{RESULTADOS}

Esta revisión de la literatura confirma que las quebradas y ríos costeros e insulares del Chocó Biogeográfico permanecen pobremente explorados, siendo pocos los inventarios faunisticos y pobre el conocimiento integral del funcionamiento de sus comunidaes y ecosistemas, a pesar de que probablemente tienen características ecológicas únicas dentro del Neotrópico, tal como lo había planteado anteriormente Blanco et al., (2009). La mayor parte de los trabajos existentes permanecen como tesis de grado profesional sin publicar. Con base en las publicaciones encontradas se hipotetiza que las quebradas costeras del Chocó biogeográfico: (1) poseen una entomofauna rica en familias y morfoespecies, pero dominada por pocos grupos cuya dinámica poblacional es modulada por los disturbios hidrológicos; (2) los ensambles de macroinvertebrados son homogéneos longitudinalmente debido a los cortos cursos; (3) los ensambles son dominados en biomasa por camarones y peces diádromos; (4) los gasterópodos diádromos están restringidos a unos pocos metros aguas arriba de las desembocaduras en los cauces que poseen doseles abiertos y que no se secan; y (5) la carcino- e ictio-fauna primariamente dulceacuícola es escasa, aunque algunas especies son endémicas.

Hipótesis 1: La entomofauna es rica en familias $y$ morfoespecies, pero dominada por pocos grupos cuya dinámica poblacional es modulada por los disturbios hidrológicos. En PNNG, Gómez-Aguirre et al., (2009) encontró diez ordenes de artrópodos (9 de Insecta y 1 de Crustacea) y 31 familias (confirmado posteriormente por Longo-Sánchez \& Blanco, 2014a en este número). Trichoptera y Ephemeroptera tuvieron el mayor número de morfoespecies. Leptophlebiidae (Ephemeroptera) fue la familia más abundante y frecuente, 
particularmente durante la temporada de lluvias. Los Chironomidae (Diptera) también fueron muy abundantes pero durante la época de menor precipitación. Por otra parte, en tres quebradas que desembocan a la bahía de Capurganá, Contreras (2012) encontró 9 órdenes y 42 familias de Insecta, siendo Ephemeroptera y Trichoptera los ordenes con mayor número de individuos (29 y 27\%, respectivamente), seguido de Diptera (13\%); mientras que Odonata y Trichoptera presentaron el mayor número de familias (8 y 7, respectivamente). En estas quebradas, aunque Leptophlebiidae (17\%) continuó siendo la familia más abundante, se encontró mayor equidad con otras familias de efemerópteros (Baetidae: 10\%; Leptohyphidae: 14\%), tricópteros (Leptoceridae: 8\%; Hydropsychidae: 14\%) y dípteros (Chironomidae: 10\%). En síntesis, El PNNG presentó una menor riqueza total, una menor abundancia y riqueza de Trichoptera, una menor abundancia de Ephemeroptera, y una menor riqueza de Odonata que en bahía Capurganá, posiblemente evidenciando el aislamiento por insularidad como un proceso importante en la colonización de las quebradas de las islas del Chocó Biogeográfico, tal como lo discutieron Longo-Sánchez \& Blanco (2009) para Gorgona y recientemente Gutierrez-Fonseca, Ramirez, Umaña \& Springer (2013) para el POT.

Estas diferencias de composición y abundancia relativa, además de estar ligadas con la insularidad o continentalidad, también pueden ser moldeadas por el régimen de precipitación (Gómez-Aguirre, 2009; Gómez-Aguirre et al., 2009). Por ejemplo, en PNNG se observaron diferencias significativas de la abundancia relativa de las familias y ordenes dominantes entre periodos de baja y alta precipitación (Ephemeroptera: 40 y 56\%, respectivamente; Trichoptera: 10 y $5 \%$, respectivamente). En bahía Capurganá no se muestreó durante el periodo de lluvias por lo tanto, no se conoce la dinámica de los grupos dominantes. Sin embargo, esta localidad recibe menor precipitación anual, con una distribución marcadamente estacional. Las quebradas pueden considerarse estacionalmente secas y algunos tramos de la parte baja de han sido observadas sin caudal por más de un año (J. F. Blanco, observ. pers.). Por lo tanto, mientras en PNNG el disturbio hidrológico lo podrían constituir las crecientes, en la ecorregión Darién el disturbio lo constituirían las sequías, particularmente en los tramos de las planicies costeras.

Hipótesis 2: Los ensambles de macroinvertebrados son homogéneos longitudinalmente debido a los cortos cursos. En PNNG, se encontró no solo una reducción de la abundancia y la riqueza de la entomofauna (GómezAguirre, 2009; Gómez-Aguirre et al., 2009), sino una homogenización longitudinal durante la época de mayor precipitación en comparación con la época de menor precipitación (Fig. 3). Esto parece ser explicado porque el aumento del caudal no solo arrastra a los individuos y sus microhábitats (e.g. gravas) a lo largo de los segmentos relativamente cortos $(1 \mathrm{~km})$ y de altas pendientes, sino que homogeniza las condiciones de los hábitats (rápidos y piscinas) en términos de profundidad y velocidad. Los substratos más gruesos como los cantos rodados parecen conferir refugios ante las crecientes, ya que presentaron una composición de morfoespecies diferente a la de las gravas, y una mayor densidad (Fig. 3). Durante el periodo de menor precipitación fueron observadas diferencias longitudinales entre tramos de las partes montañosa y plana, pero no entre microhábitats (Fig. 3). Las diferencias longitudinales parecen ser más prevalentes en la ecorregión Darién con el incremento del régimen de la sequía (Contreras, 2012).

Hipótesis 3: Los ensambles son dominados en biomasa por camarones y peces diádromos. Las especies diádromas, son dulceacuícolas secundarias, de origen marino, que se desarrollan como huevos y larvas en aguas marinas o salobres y posteriormente migran río arriba como postlarvas y juveniles para establecerse como adultos y reproducirse. Estos adultos usualmente presentan una tolerancia limitada a aguas salobres o marinas y se pueden distribuir extensamente a lo largo de la cuenca. 


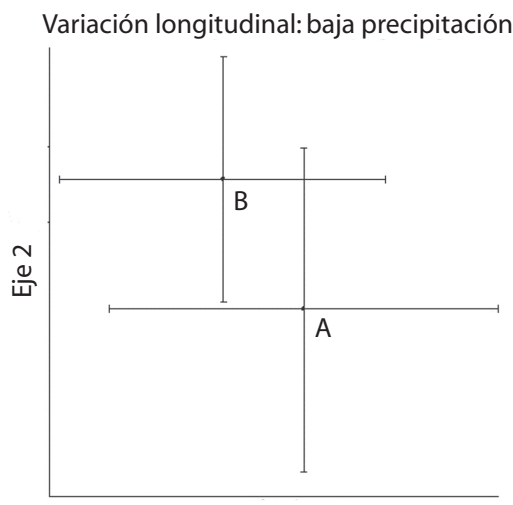

Eje 1

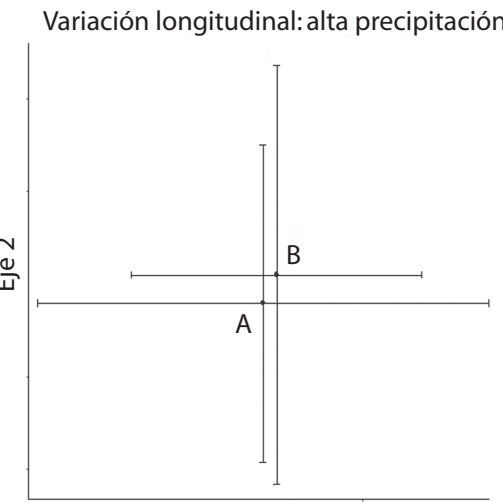

Eje 1

Variación entre substratos: baja precipitación Variación entre substratos: alta precipitación

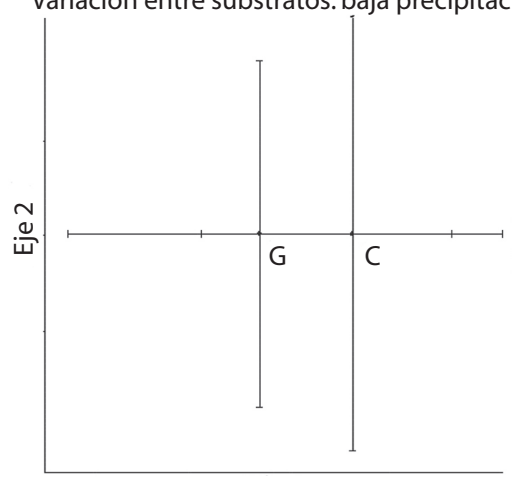

Eje 1

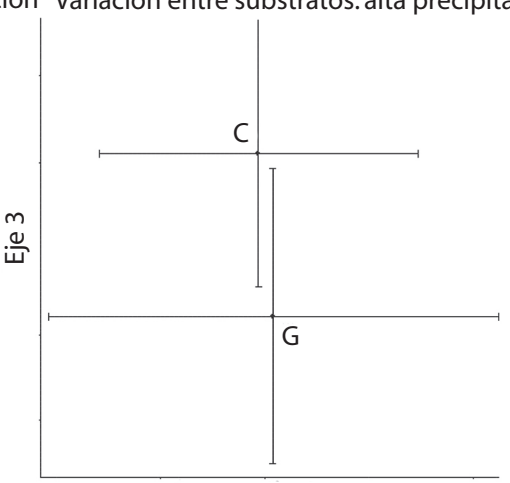

Eje 1

Fig. 3. Ordenación de la composición de morfoespecies de macroinvertebrados utilizando un Escalamiento no Métrico Multidimensional (NMDS, sigla en inglés). Se utilizaron 104 unidades de muestreo durante un periodo de baja precipitación y 92 durante uno de alta, en varias quebradas alrededor del PNNG. El estrés del modelo es 16.25 para las ordenaciones durante baja precipitación y 16.53 para las ordenaciones de alta precipitación. Se agruparon las unidades de muestreo con respecto al tramo (A: alto, B: bajo) y el tipo de substrato (C: cantos rodados, G: gravas). Durante el periodo de alta precipitación no observaron diferencias significativas del ensamblaje entre los tramos, pero si entre tipos de substrato. Procedimiento utilizado: Permutaciones de Múltiples Respuestas (MRPP, sigla en inglés) en PC Ord. El punto representa las coordenadas promedio de cada grupo y las cruces representan la desviación estándar.Modificado de: Gómez-Aguirre, 2009. Fig. 3. Ordination of the macroinvertebrate morphospecies composition using a Non-Metric Multi-Dimensional Scaling (NMDS). Sampling units: 104 during the low rainfall period and 92 during the high rainfall period, collected in several streams around Gorgona NNP. Model stress: 16.25 for low rainfall period, and 16.53 for high rainfall period. Sampling units were grouped according to reach position (A: high, B: low), and substrate type (C: cobble, G: gravel). No significance difference was observed between reaches during the high rainfall period, but significant differences were observed between substrate types during the same period. The tests were performed using the Multiple Response Permutation Procedure (MRPP) in PC Ord. The mean coordinates are shown with a dot, and the standard deviations with the cross. Modified from: Gómez-Aguirre, 2009.

Los dos tipos de diadromía más frecuentes en las quebradas tropicales son la catadromía y la anfidromía (McDowall, 2007).

Prahl, Guhl \& Grogl (1979), Prahl, Caicedo \& Ríos (1984) reportaron varias especies de palaemonidos en quebradas y ríos a lo largo del Pacífico colombiano. En las quebradas del PNNG y de bahía Capurganá son observadas a simple vista varias especies de camarones diádromos del género Macrobrachium (Palaemonidae; muchillá) (Fig. 4, Cuadro 2). Aunque la captura de los adultos requiere el 

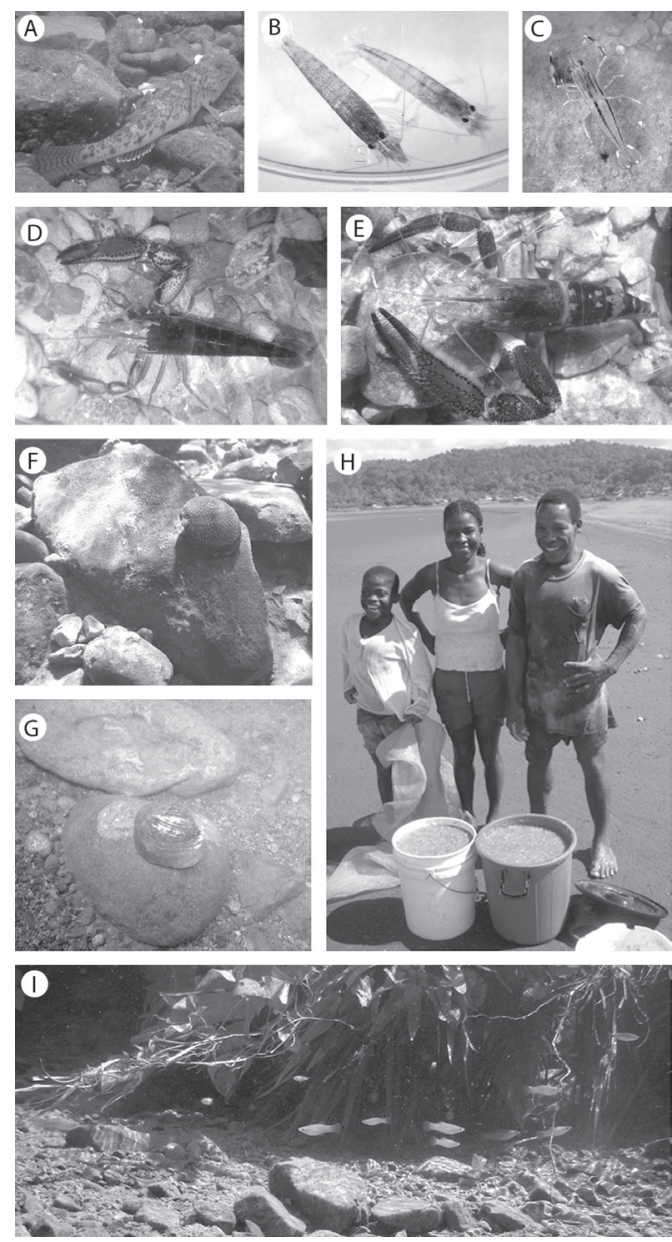

Fig. 4. Fauna típica de las quebradas costeras del Chocó Biogeográfico (Colombia): A) Bathygobius sp. (Gobiidae) de PNN Gorgona, similar a Awous tajasica (Gobiidae) del Caribe. B) Potimirin glabra (Atyidae) colectado en PNN Gorgona y ecorregión Darién. C y D) Macrobrachium americanum y $M$. hancocki (Palaemonidae) en PNN Gorgona, respectivamente. E) M. crenulatun (Palaemonidae) en río Capurganá (ecorregión Darién). F y G) Gasterópodos Neritidae (N. punctulata en río Capurganá y $N$. latissima en PNN Gorgona, respectivamente). H) Baldes con larvas de Gobiidae capturadas durante un evento de migración río-arriba en masa (fenómeno "La Viuda") en la desembocadura del río El Valle (ecorregión Baudó). I) Poecilidae en el río Capurganá. Fotos de los autores, excepto B.

Fig. 4. Representative fauna of coastal streams in the Biogeographic Chocó (Colombia): A) Bathygobius sp. (Gobiidae) in Gorgona NNP, similar to Awous tajasica (Gobiidae) in the Caribbean. B) Potimirin glabra (Atyidae) collected in Gorgona NNP and Darién ecoregion. C and D) Macrobrachium americanum and $M$. hancocki (Palaemonidae) in Gorgona NNP, respectively. E) $M$. uso de trampas y cebos, en PNNG se colectaron frecuentemente juveniles con redes Surber (Gómez-Aguirre et al., 2009; Longo-Sánchez \& Blanco, 2014a en este número). Además en muestras de hojarasca de ambas localidades se colectaron Potimirin cf. glabra (Atyidae) (Fig. 4) (Longo-Sánchez \& Blanco, 2014b en este número; Escobar-Sierra \& Blanco datos sin publicar). La biomasa per capita de estas especies de camarones, aun las de pequeño tamaño corporal como $P$. glabra, exceden la biomasa total y per capita de los insectos con excepción de las cucarachas (Longo-Sánchez $\&$ Blanco, 2014b en este número).

Además de los camarones, varias especies de peces diádromos han sido observadas en las quebradas costeras del Chocó Biogeográfico. En la región es abundante la lisa de montaña o nayo, Agonostomus monticola (Mugilidae) (Fig. 4), la cual tiene una distribución anfipanámica $\mathrm{y}$ ha sido colectada en el PNNG (Cala, 1990; J.F. Blanco, observ. pers.), y en varios ríos de las ecorregiones Baudó (ríos Valle, Baudó, Juradó y Jurubidá: Fowler, 1944; Carvajal-Quintero, 2011) y Darién (ríos Acandí y Capurganá: Álvarez-León, 1991; C. EscobarSierra \& J. F. Blanco, datos sin publicar). En el PNNG y las ecorregiones Baudó y Darién también se han reportado otras especies diádromas de las familias Eleotridae y Gobiidae (Fig. 4, Cuadro 2). Aunque se conoce coloquialmente que en algunas localidades del Chocó Biogeográfico se presentan migraciones río arriba en masa de larvas de camarones y peces diádromos, tal como ha sido reportado para otras partes del mundo, Carvajal-Quintero (2011), Castellanos-Galindo, Sanchez, BeltránLeón \& Zapata (2011), y Sánchez-Garcés,

crenulatun (Palaemonidae) in Capurganá River (Darién Ecoregion).F y G) Neritidae gastropods ( $N$. punctulata in Capurganá River and N. latissima in Gorgona NNP, respectively). H) Buckets with Gobiidae larvae collected during a massive upstream migration (locally known as "La Viuda") in El Valle River estuary (Baudó Ecoregion). I) Poecilidae in Capurganá River. Photos taken by the authors, except B. 
CUADRO 2

Especies de peces y camarones diádromos presentes en las quebradas costeras del Chocó Biogeográfico.

TABLE 2

Species of diadromous fish and shrimp present in coastal streams in the Biogeographic Chocó.

\begin{tabular}{|c|c|c|}
\hline Ecorregión & Especie (Familia) & Localidades (Fuente) \\
\hline Darién & $\begin{array}{l}\text { Agonostomus monticola } \text { Bancroft, } 1834 \text { (Mugilidae) } \\
\text { Mugil curema } \text { Valenciennes, } 1836 \text { (Mugilidae) } \\
\text { Gobiomorus dormitor Lacepède, } 1800 \text { (Eleotridae) } \\
\text { Eleotris pisonis Gmelin, } 1789 \text { (Eleotridae) } \\
\text { Awaous tajasica (Lichtenstein, 1822) (Gobiidae) } \\
\text { Sicydium plumieri Bloch, } 1786 \text { (Gobiidae) } \\
\text { Macrobrachium americanum Bate, } 1868 \text { (Palaemonidae) } \\
\text { Macrobrachium acanthurus Wiegmann, } 1836 \text { (Palaemonidae) } \\
\text { Macrobrachium carcinus Linée, } 1758 \text { (Palaemonidae) } \\
\text { Macrobrachium crenulatum Holthius, } 1950 \text { (Palaemonidae) } \\
\text { Potimirin glabra Kingsley, } 1978 \text { (Atyidae) }\end{array}$ & $\begin{array}{l}\text { Río Acandí, río Capurganá, Sapzurro, San } \\
\text { Francisco y quebradas aledañas } \\
\text { (Álvarez-León, 1991; Triana \& Campos, } \\
\text { 2007; Escobar-Sierra \& Blanco, observ. } \\
\text { pers. 2010-2011). }\end{array}$ \\
\hline Baudó & $\begin{array}{l}\text { Agonostomus monticola (Mugilidae) } \\
\text { Gobiomorus maculatus Günther, } 1859 \text { (Eleotridae) } \\
\text { Eleotris picta Kner, } 1863 \text { (Eleotridae) } \\
\text { Hemieleotris latifasciata } \text { Meek \& Hildebrand, } 1912 \text { (Eleotridae) } \\
\text { Dormitator latifrons } \text { Richardson, } 1844 \text { (Eleotridae) } \\
\text { Awaous banana } \text { Valenciennes, } 1837 \text { (Gobiidae) } \\
\text { Sicydium salvini Ogilvie-Grant, } 1884 \text { (Gobiidae) } \\
\text { Macrobrachium americanum Bate, } 1868 \text { (Palaemonidae) } \\
\text { Macrobrachium transandicum (Holthius, 1950) }\end{array}$ & $\begin{array}{l}\text { Río Juradó, río Valle, río Jurubidá, río } \\
\text { Baudó, Nuquí (Fowler, 1944; Valencia \& } \\
\text { Campos, 2007; Carvajal-Quintero, 2011). }\end{array}$ \\
\hline Gorgona & $\begin{array}{l}\text { Agonostomus monticola Bancroft, } 1834 \text { (Mugilidae) } \\
\text { Gobiomorus sp.(Eleotridae) } \\
\text { Bathygobious sp.(Gobiidae) } \\
\text { Tomicodon myersi Briggs, } 1955 \\
\text { Macrobrachium hancockii Holthius, } 1950 \text { (Palaemonidae) } \\
\text { Macrobrachium panamense Rathbun, } 1912 \text { (Palaemonidae) } \\
\text { Macrobrachium americanum Bate, } 1868 \text { (Palaemonidae) } \\
\text { Macrobrachium rathbunae Holthius, } 1950 \text { (Palaemonidae) } \\
\text { Potimirin glabra Kingsley, } 1978 \text { (Atyidae) }\end{array}$ & $\begin{array}{l}\text { Varias quebradas (Rubio, 1986; Aguirre } \\
\text { \& Rangel, 1990; Cala, 1990; Valencia } \\
\text { \& Campos, 2007; Blanco, 2010-2011 } \\
\text { observ. pers.). }\end{array}$ \\
\hline
\end{tabular}

Castellano-Galindo, Beltrán-León \& Zapata. (2011) recientemente informaron sobre este fenómeno denominado "La Viuda" en el río Valle al norte de la ensenada de Utría en la ecorregión Baudó, dominado numéricamente por larvas de Gobiidae (Fig. 4).

Hipótesis 4: Los gasterópodos diádromos están restringidos a unos pocos metros aguas arriba de las desembocaduras en los cauces que poseen doseles abiertos y que no se secan. En PNNG se encontró a Neritina latissima (Prosobranchia: Neritidae) (GómezAguirre et al., 2009) y en la ecorregión Darién (i.e. quebrada Capurganá) a N. punctulata (J. F. Blanco, observ. pers.) (Fig. 4) en muy bajas densidades $\left(<1\right.$ ind. $\left./ \mathrm{m}^{2}\right)$, restringidas a unas decenas de metros aguas arriba de las desembocaduras. En el PNNG se hipotetiza que no es más abundante debido a que los doseles cerrados sobre los tramos de las planicies costeras limitan la producción perifítica que sostiene a estos herbívoros, y por otro lado la alta frecuencia de crecientes causa alta mortalidad o desplazamiento hacia las desembocaduras. En la ecorregión Darién se hipotetiza que la alta frecuencia de sequías, deseca extensos tramos de la planicie costera, causando la 
muerte de los pocos individuos que logran subir por el río durante los periodos de lluvias. Estas observaciones contrastan con lo encontrado en ríos de órdenes mayores en ambas ecorregiones donde se han hayado individuos varios kilómetros arriba de la desembocadura (Pacífico-río Dagua: García, Cantera, Zuñiga \& Montoya, 2009; Caribe-río Atrato: J. F. Blanco, observ. pers.).

Hipótesis 5: La carcino e ictio-fauna primariamente dulceacuícola es escasa, aunque algunas especies son endémicas. En las quebradas costeras del Chocó Biogeográfico, las especies dulceacuícolas primarias presentes son de origen andino (Fowler, 1944; Cala, 1990; Álvarez-León, 1991; Fernández \& Schaeffer, 2005; Abell et al., 2008). Desde la parte media hasta las cabeceras de las quebradas y ríos costeros de mayor caudal (Juradó, Jurubidá, Baudó, Valle, Acandí) de las ecorregiones Baudó y Darién se han colectado varias especies y familias de Characiformes, Siluriformes, Cyprinodontiformes, Gymnotiformes y Perciformes (Cuadro 3, Fowler, 1944; ÁlvarezLeón, 1991), las cuales han sido reportadas en otras cuencas andinas. La orogénesis de las serranías de Baudó y Darién puede explicar la relativa alta riqueza y alto grado de endemismo de especies de peces dulceacuícolas primarias en los segmentos altos y medios de los ríos costeros del Chocó Biogeográfico (Fowler, 1944). Se propone que la formación de estos cuerpos montañosos cerca al litoral confinó a estas especies dentro de las cuencas costeras y las sometió a un alto grado de aislamiento. Infortunadamente, no existen inventarios ícticos de quebradas costeras de pequeño orden en el Chocó Biogeográfico pero se hipotetiza que debido al alto régimen de disturbio hidrológico de crecientes o sequías tengan pocas especies primariamente dulceacuícolas, probablemente endémicas, tal como sucede en el PNNG. En las quebradas de la bahía Capurganá solamente se han encontrado representantes primariamente dulceacuicolas, a algunos Poecilidae dentro de los charcos remanentes en los tramos secos de la planicie costera y algunos Cichlidae en
CUADRO 3

Familias de peces primariamente dulceacuícolas encontrados en la ecorregión Baudó (Pacífico norte colombiano). (Fowler, 1944; Carvajal-Quintero, 2011).

TABLE 3

Families of primary freshwater fishes found in the Baudó Ecorregion (Northern Pacific Coast of Colombia). (Fowler, 1944; Carvajal-Quintero, 2011).

\begin{tabular}{ll}
\multicolumn{1}{c}{ Orden } & \multicolumn{1}{c}{ Familia } \\
Characiformes & Curimatidae \\
& Characidae \\
& Erythrinidae \\
& Lebiasinidae \\
& Cetopsidae \\
Siluriformes & Trichomycteridae \\
& Callichthyidae \\
& Astroblepidae \\
& Loricariidae \\
& Heptapteridae \\
& Sternopigidae \\
Gymnotiformes & Hypopomidae \\
& Apteronotidae \\
Cyprinodontiformes & Rivulidae \\
& Poeciliidae \\
Synbranchiformes & Synbranchidae \\
Perciformes & Cichlidae \\
\hline
\end{tabular}

los tramos montañosos (C. Escobar-Sierra \& J. F. Blanco, datos sin publicar).

En el PNNG, recientemente se describió un pez endémico, dulceacuícola primario, Trychomicterus gorgonae (Trychomicteridae) (Fernández \& Schaeffer, 2005), el cual, sumado al cangrejo anfíbio Hippolobocera gorgonae (Pseudothelphusidae), también endémico de la Isla (Prahl, 1983), sugiere la relación biogeográfica con especies dulceacuícolas primarias típicas de las quebradas de cabeceras andinas de la cordillera Occidental. De hecho, la ocurrencia de estas especies apoya la hipótesis de que la isla Gorgona está situada sobre la cuarta cordillera andina (actualmente sumergida) que se desprende desde Ecuador y que hizo parte del antiguo valle del río Patía durante la última glaciación (Kerr, 2005). Dado que no existe ninguna otra especie primariamente dulceacuícola compartida con el continente, se sugiere 
que esta fauna es producto de una distribución ancestral y no de un proceso de colonización reciente sobre la estrecha barrera impuesta por el mar (35-54km).

\section{DISCUSIÓN}

\section{Composición de los ensamblajes de macroinvertebrados y las discrepancias con el Concepto del Río Continuo (CRC): Las} quebradas costeras del Chocó Biogeográfico, aun las insulares del PNNG, presentan una alta riqueza de familias y la ocurrencia de todos los órdenes de Insecta, lo cual las hace similares a las quebradas epicontinentales andinas (que no desembocan directamente en el mar) ubicadas en la zona de vida de selva húmeda tropical. En el PNNG, esta alta riqueza se ha explicado por la cercanía a la costa Pacífica colombiana ( $35 \mathrm{~km}$ a la parte más cercana) y en la bahía Capurganá se explica por su posición continental dentro de una ecorregión de alta biodiversidad como Darién. En las quebradas del arco antillano se ha encontrado que aquellas ubicadas más cerca a Suramérica presentan un mayor número de especies (Bass 2003). La composición de morfoespecies y estructura (dominancia de pocas especies) del ensamblaje es similar a la encontrada por Boyero \& Bailey (2001) en una quebrada de la isla de Coiba (Panamá) y a la reportada para otras quebradas continentales tropicales (sintetizado por Jacobsen et al., 2008). Recientemente se publicó que las islas del POT se ajustan a las predicciones de la teoría de la biogeografía de islas (Gutierrez-Fonseca et al., 2013)

La dominancia de Leptophlebiidae (Ephemeroptera) en PNNG y la codominancia de otros Trichoptera en la ecorregión Darién pueden reflejar un gradiente sur-norte de influencia de las crecientes. En PNNG, y posiblemente en la ecorregión Baudó, las frecuentes crecientes parecen ser el disturbio predominante que filtra especies, de tamaños corporales grandes, poca movilidad y ciclos de vida largos, mientras que en la ecorregión Darién la ausencia de crecientes durante varios meses e incluso años permite la colonización de grupos de condiciones más estables como los tricópteros. Se conoce que varios grupos de efemerópteros neotropicales, son abundantes y frecuentes en climas lluviosos y presentan un comportamiento derivador constante a lo largo del año (e.g. Leptophlebiidae, Baetidae, Leptohyphidae en Costa Rica: Ramírez \& Pringle, 1998a; 1998b). En general, las crecientes son un factor controlador de la dinámica de las comunidades de insectos de quebradas neotropicales, ya que reduce la riqueza y la densidad, y homogeniza la composición taxonómica durante los periodos de lluvia (Flecker \& Feifarek, 1994; Ramírez \& Pringle, 1998b; Ramírez et al., 2006). En PNNG, también se encontró que la composición taxonómica se homogenizaba entre tramos montañosos y de planicie durante el periodo de lluvias, principalmente por el arrastre de los individuos y especies más sensibles y por la corta extensión de los cursos (Gómez-Aguirre, 2009). Los substratos gruesos (bloques y cantos rodados), y por lo tanto la litología de la cuenca (basáltica vs. sedimentaria), parecen jugar un papel importante en la provisión de refugios (Gómez-Aguirre, 2009; Longo-Sánchez \& Blanco, 2014a en este número).

Los anteriores hallazgos sugieren que en quebradas suficientemente cortas del Chocó Biogeográfico, las diferencias en composición de especies equivalentes a las propuestas por el CRC (Vannote et al., 1980) son débiles o desaparecen durante la época de crecientes. Sin embargo, durante el periodo de menor precipitación podrían emerger, pero principalmente en climas más secos como los del norte del Chocó Biogeográfico y el Caribe. Las diferencias longitudinales parecen ser más prevalentes en la ecorregión Darién con el incremento del régimen de la sequía, tal como se ha observado en quebradas del Caribe donde algunos grupos de fauna diádroma como los caracoles desaparecen (Blanco \& Scatena, 2006).

Uno de los postulados más importantes del CRC es el cambio longitudinal de las redes tróficas en función de la apertura de dosel. Las quebradas de bajo orden $(<3)$ del Chocó Biogeográfico usualmente desembocan en el mar por debajo de doseles cerrados, 
evitando incrementos significativos en la producción perifítica y promoviendo un gran aporte de hojarasca. Adicionalmente, los principales detritívoros son los camarones, los cuales se encuentran ampliamente distribuidos a lo largo de los cursos, lo cual contradice el postulado del CRC en el cual los detritívoros (principalmente insectos) se concentran en las cabeceras. Esto fue advertido varias décadas atrás para las quebradas de Nueva Zelanda por Winterbourn et al. (1981).

Recientemente, Greathouse \& Pringle (2006), en su evaluación rigurosa del CRC, propusieron que las quebradas de bajo orden en Puerto Rico, aun de varios kilómetros de largo, no eran lo suficientemente retentivas por lo tanto la respiración excedía la producción primaria a lo largo de todo el curso. También, encontraron que básicamente se podían distinguir dos zonas relacionadas con el gradiente y la dominancia de la fauna: cabecera, dominada por camarones, y planicie, dominada por caracoles, ambas con baja abundancia y biomasa de insectos. Estudios más recientes en otras islas del Caribe han encontrado una homogeneidad de las comunidades de insectos a lo largo del curso de los ríos, siendo sólo discernibles las partes bajas (por la adición de caracoles nerítidos) y las cabeceras (por la adición algunas especies de cangrejos e insectos), y variaciones locales influenciadas por los cambios en la cobertura vegetal ribereña, y por lo tanto la variación longitudinal del ensamblaje de macroinvertebrados se desvía de las predicciones del CRC y otros sistemas de zonación europeos basados en la temperatura de las aguas (Trinidad: Turner et al., 2008; Jamaica: Hyslop \& Hunte-Brown, 2012).

Por lo tanto, se propone someter a prueba la aplicabilidad de otros conceptos diferentes al CRC tales como la discontinuidad serial y las zonas de estrés hidráulico. Las cascadas imponen barreras para la distribución de los camarones y peces diádromos, ocasionando diferencias en la estructura de las comunidades de insectos y el funcionamiento del ecosistema aguas arriba y aguas abajo. Por otra parte, las pequeñas cascadas y las discontinuidades geomorfológicas causadas por la discontinuidad litología (roca madre expuesta) causa zonas de estrés hidráulico que pueden causar diferencias en la estructura de las comunidades y el funcionamiento del ecosistema decenas o cientos de metros aguas abajo. Finalmente, se sugiere explorar más el papel del disturbio de crecientes en las zonas de alta precipitación, ya que podría estar previniendo la estructura de las comunidades en condiciones de equilibrio. En el norte del Chocó Biogeográfico y el Caribe colombiano, valdría la pena explorar el otro extremo de disturbio impuesto por la sequía, la cual se constituye un factor selectivo por adversidad. Por lo tanto, el gradiente de condiciones climáticas sur-norte permite someter a prueba diferentes paradigmas de organización de las comunidades y ecosistemas lóticos, propuestos para quebradas de zonas templadas, y no simplemente aplicarlos como lo advierten Boulton et al. (2008).

Finalmente, para someter a prueba de manera robusta la teoría ecológica es necesario fortalecer la resolución taxonómica de muchos grupos de insectos tanto en Colombia como en el Neotrópico. Por ejemplo, los pocos estudios taxonómicos disponibles para Chironomidae y Simulidae han mostrado que son ricos en géneros y especies (Coffman \& de la Rosa, 1998; Ramirez \& Pringle, 2006; Moncada, Mantilla, Lotta, Matta \& Adler, 2013). Estos grupos por su abundancia y diversidad podrían permitir análisis estadísticos, espaciales y bionómicos más robustos en comparación a otros grupos raros, para someter a prueba diferentes hipótesis ecológicas. Finalmente, métodos de muestreo específicos para inmaduros, exhuvias y adultos podrían permitir abordar hipótesis específicas a diferentes escalas espaciales.

Influencias ecológicas propagadas aguas arriba mediadas por la fauna diádroma: $\mathrm{La}$ exploración ictiológica en Colombia ha sido la principal responsable del avance del conocimiento ecológico de los ríos y quebradas. Sin embargo, dicha actividad se ha concentrado en la zona andina (Maldonado-Ocampo et al., 2005) y muy poco se han abarcado las zonas 
costeras tanto del Pacífico y como del Caribe (e.g. Álvarez-León, 1991; Agudelo-Zamora, Ospina-Pabón \& Jiménez-Segura, 2010). Este vacío de información es aún mayor en los sistemas de bajo orden. Sin embargo, las pocas exploraciones sintetizadas en esta revisión y nuestra propia experiencia permiten hipotetizar que las quebradas y ríos costeros del Chocó Biogeográfico podrían ajustarse a modelos ecológicos mejor estudiados en otras partes dentro del Trópico como son las islas oceánicas volcánicas (Macioleck \& Ford, 1987; Resh \& De Szalay, 1995), caracterizadas por: (1) drenajes cortos que corren sobre sustratos basálticos dominados por roca madre, bloques y cantos rodados; (2) cuencas con coberturas vegetales de gran densidad que aportan constantemente materia orgánica; (3) un régimen de caudal variable que actúa como un impacto considerable para sus comunidades; y (4) ensamblajes de consumidores dominados por especies diádromas. Estas últimas en el Chocó Biogeográfico tienen una composición de especies o géneros similar a la de ríos del Caribe centroamericano y Las Antillas. Por ejemplo, varias especies de camarones Macrobrachium spp. y Potimirin glabra, y peces (A. monticola, S. plumieri, A. tajasica) encontradas en quebradas y ríos costeros del Caribe centroamericano (Lorion, Kennedy \& Braatne, 2011) y Las Antillas (Fievet, Doledec \& Lim, 2001; Hein et al., 2011) han sido encontradas en muestreos en la ecorregión Darién (Álvarez-León, 1991; Agudelo-Zamora et al., 2010; C. Escobar-Sierra \& J. F. Blanco, observ. pers.) y aun en las ecorregiones Baudó y Gorgona (A. monticola y P. glabra: Cala, 1990; Gómez-Aguirre et al., 2009; Longo-Sánchez \& Blanco 2014a; 2014b; J. D. CarvajalQuintero y J. F. Blanco, observ. pers.). También las quebradas del Pacífico colombiano comparten grupos de peces y gasterópodos con Costa Rica (Lyons \& Schneider, 1990; Schneider \& Lyons, 1993).

Debido a que todos estos grupos son presumiblemente diádromos, y en algunos casos migraciones masivas han sido observadas (peces: Keith, 2003, Bell, 2007; CarvajalQuintero, 2011; caracoles: Blanco \& Scatena,
2005; 2007; camarones: Kikkert \& Crowl, 2009; Carvajal-Quintero, 2011), su distribución a lo largo de las quebradas costeras promueve importantes controles sobre la estructura de las comunidades de macroinvertebrados (principalmente insectos) y el funcionamiento del ecosistema (e.g. producción primaria) (Pringle, 1997; Ramírez \& Hernández-Cruz, 2004; Hein et al., 2011). Debido a que la distribución de esta fauna diádroma es controlada principalmente por la presencia de cascadas y tramos de alta pendiente, la abundancia, biomasa y riqueza de insectos y camarones presentan discontinuidades aguas arriba y aguas debajo de estas geoformas (Pringle, 1997; Ramírez \& Hernández-Cruz, 2004; Hein et al., 2011). Por esta razón la investigación ecológica en las quebradas costeras del Chocó Biogeográfico debe centrarse inicialmente en establecer el papel estructurador de esta fauna y sobre "la propagación de sus influencias aguas arriba" (sensu Pringle, 1997). Finalmente, las quebradas y ríos costeros de órdenes mayores con presencia de peces primariamente dulceacuícolas podrían asemejarse funcionalmente a los sistemas más grandes de Cuba y La Española (C. Santos, Universidad de Puerto Rico, Mayaguez, com. pers.). Se propone estudiar la auto- y sin-ecología de esta ictiofauna, particularmente para establecer el grado de competencia con la ictiofauna diádroma y su papel en la estructura de las comunidades y los procesos ecosistémicos.

Implicaciones para las redes tróficas acuáticas y procesos ecosistémicos: Los grandes aportes de hojarasca determinados por los doseles cerrados en los tramos de pequeño orden (Valencia et al., 2009; Longo-Sánchez \& Blanco, 2014b en este número) sugieren que los detritos son los principales recursos para los macroconsumidores. De hecho, se ha establecido que los camarones son los principales responsables del fraccionamiento de la hojarasca en quebradas insulares del Caribe (Pringle, Hemphill, McDowell, Bednarek \& March, 1999; Crowl, McDowell, Covich \& Johnson, 2001) y por lo tanto su papel en el 
Chocó Biogeográfico podría ser similar. Adicionalmente, Longo-Sánchez \& Blanco (2014b en este número) encontraron que las cucarachas (Blaberidae: Epilampra) y otros insectos, además de los camarones Potimirin glabra, son los detritívoros dominantes en las quebradas del PNNG, lo cual pone al Chocó Biogeográfico dentro de la discusión global de la importancia de los detritívoros en el proceso de descomposición. Estos controles desde el tope de las redes tróficas (top-down controls) son los principales responsables de las desviaciones de las predicciones del CRC en quebradas de Puerto Rico (Greathouse \& Pringle, 2006). Finalmente, a pesar de las grandes similitudes de los ensamblajes de fauna diádroma y fisonomía de las quebradas costeras del Chocó Biogeográfico con las de Centroamérica y Las Antillas, su carácter más distintivo es la baja densidad o ausencia generalizada de gasterópodos nerítidos, los cuales son los principales ramoneadores en las quebradas de doseles abiertos en otras regiones tropicales donde alcanzan altas densidades y biomasas (Blanco \& Scatena, 2006; Greathouse \& Pringle, 2006).

Influencias ecológicas desde las cabeceras: A pesar de las conexiones ecológicas entre la parte baja de las quebradas costeras y sus cabeceras establecidas por la fauna diádroma, existen influencias geomorfológicas e hidrológicas impuestas por las cabeceras en estas quebradas de manera equivalente a lo que sucede con las quebradas en montañas más altas de zonas templadas y tropicales (Gomi, Sidle \& Richardson, 2002; Scatena \& Gupta, 2012). Se recomienda estudiar el papel de los derrumbes en la alteración de los cauces, los substratos y los aportes de madera y hojarasca, ya que son procesos frecuentes debido a las altas precipitaciones (J. F. Blanco, observ. pers.). También dependiendo del origen geológico y del aspecto de las cuencas, la química de las aguas puede ser diferente, evidenciada en el pH y la conductividad, lo cual se ha encontrado como un factor estructurador importante en paisajes volcánicos de Centroamérica (Ramírez et al., 2006), y posiblemente también en el PNNG (Blanco, 2009b; Gómez-Aguirre, 2009; Gómez-Aguirre et al., 2009; Longo-Sánchez \& Blanco, 2014a en este número). También el grado de desarrollo de los bosques ribereños en las cabeceras tiene influencia directa sobre las cantidades y cualidades de la hojarasca que se acumula en las quebradas (PNNG: Valencia et al., 2009). Finalmente, las características geomorfológicas de las cabeceras en interacción con el régimen de precipitación pueden promover regímenes de crecientes diferentes los cuales también tienen una influencia distintiva sobre las comunidades y procesos ecosistémicos lóticos.

En conclusión, se sugiere que la mayor parte de las quebradas costeras del Chocó Biogeográfico, ubicadas en el continente o en islas, no se ajustan a los postulados del CRC debido a que éstas poseen características similares a las islas volcánicas del Pacífico y el Caribe, tales como drenajes cortos, regímenes de caudales torrenciales, doseles cerrados, y dominancia de especies diádromas. Esta última característica podría determinar la estructura de las comunidades de insectos y la dinámica de procesos ecosistémicos. Sin embargo, las quebradas de esta provincia biogeográfica parecen tener características particulares tales como una frecuente tasa de disturbios de creciente en la vertiente Pacífico y frecuente sequía en la vertiente Caribe. En las quebradas y ríos de ordenes mayores continentales e insulares hay ocurrencia de varias especies de peces dulceacuícolas primarios, algunas de ellas endémicas. En las quebradas más pequeñas hay una ausencia funcional de caracoles, al igual que de peces primariamente dulceacuícolas. Finalmente, la entomofauna es rica pero aparentemente afectada por procesos de insularidad y disturbio hidrológico (crecientes y sequías). La síntesis de estas características permitirá una mejor comprensión de la ecología de estos sistemas. También permitirá utilizarlos como modelos para estudio ecológicos de quebradas costeras e insulares, y como lugares para someter a prueba hipótesis ecológicas generales teniendo en cuenta las similitudes y las diferencias con otras partes del mundo donde fueron propuestas originalmente. Aunque las quebradas del 
PNNG representan las generalidades geomorfológicas, hidrológicas y de la biota diádroma de las quebradas costeras del Chocó Biogeográfico y de las islas oceánicas volcánicas, su ubicación sobre la plataforma continental le confiere una composición y estructura de la entomofauna intermedias entre los extremos oceánico y continental, haciéndolas diferentes, requiriendo que las extrapolaciones de los estudios desde y hacia la Isla se hagan con cuidado.

\section{AGRADECIMIENTOS}

El trabajo de campo se realizó dentro de los proyectos: "Gorgona Island Stream Bioassessment" financiado por el CODI de la Universidad de Antioquia y autorizado por el permiso de investigación DTSO-G-03/08 de la Unidad Administrativa del Especial del Sistema de Parques Nacionales Naturales (UAESP$\mathrm{NN}$ ), "Aportes al plan de manejo pesquero en el PNN Utría" financiado por la UAESPNN, Patrimonio Natural y la Universidad de Antioquia (Convenio 017/021), "Expedición Estuarina, golfo de Urabá" financiado por la Gobernación de Antioquia y la Universidad de Antioquia, y «Rasgos biológicos de los insectos acuáticos como respuesta a la perturbación natural por sequía en los ríos y quebradas de la ecoregión Capurganá, Chocó Biogeográfico, Municipio de Acandí» financiado por el CODI de la Universidad de Antioquia, el Instituto de Estudios Ambientales del Pacífico (IAP), la Asociación Nacional de Estudiantes de Biología (ANEB) y el Consejo Comunitario Negro del Río Acandí y Chocó Norte. La investigación en Puerto Rico ha sido apoyada por el Programa de Investigación Ecológica a Largo Plazo en el Bosque Experimental de Luquillo financiado por la Fundación Nacional de Ciencias de los Estados Unidos de Norteamérica. Se agradece a Pilar X. Lizarazo por ceder la foto 4B. Contribución ELICE No. 9.

\section{RESUMEN}

A pesar de la escasez de estudios sobre la geología, hidrología y biota de las quebradas costeras ubicadas en el
Chocó Biogeográfico, se hipotetiza que éstas se ajustan a la tipología observada en las islas oceánicas volcánicas del Caribe y el Pacífico, las cuales no cumplen con las predicciones del Concepto del Río Continuo. Estas quebradas son cortas $\left(<10^{1} \mathrm{~km}\right)$ y muy pendientes debido a su origen tectónico, y presentan lechos dominados por bloques y cantos rodados. El régimen de caudal es torrencial en la vertiente Pacífico, pero estacionalmente seco en la vertiente Caribe. Los doseles cerrados aportan grandes cantidades de hojarasca a las quebradas que desembocan al mar con bajo orden. Las especies diádromas, con afinidades centroamericanas y antillanas, dominan los ensamblajes y posiblemente cumplen papeles ecológicos equivalentes a los establecidos en otras regiones. Particularmente, las quebradas costeras de esta provincia presentan varias especies de peces dulceacuícolas primarios, algunas endémicas, y carecen de gasterópodos. Los procesos geomorfológicos e hidrológicos de las cabeceras influyen sobre la dinámica ecosistémica de estas quebradas. Se proponen cinco hipótesis sobre la estructura y composición comunitaria. Se advierte que el PNNG es un modelo útil para la ecología lótica costera, pero que tiene particularidades.

Palabras clave: quebradas costeras, Neotrópico, fauna diádroma, insectos acuáticos, ecología lótica, Concepto del Río Continuo.

\section{REFERENCIAS}

Abell, R., Thieme, M. L., Revenga, C., Bryer, M., Kottelat, M., Bogutskaya, N., Coad, B., Mandrak, N., Contreras-Balderas, S., Bussing, W., Stiassny, M. L. J., Skelton, P., Allen, G. R., Unmack, P., Naseka, A., Ng, R., Sindorf, N., Robertson, J., Armijo, E., Higgins, J. V., Heibel, T. J., Wikramanayake, E., Olson, D. López, H. L., Reis, R. E., Lundberg, J. G., Sabaj-Pérez, M. H. \& Petry, P. (2008). Freshwater ecoregions of the World: A new map of biogeographic units for freshwater biodiversity conservation. Bioscience, 58: 403-414.

Agudelo-Zamora, H. D., Ospina-Pabón, J. G. \& JiménezSegura, L. F. (2010). Peces del río San Juan de Urabá, costa Caribe, Colombia, Sur América. Bol. Cien. Centro Museos, Museo de Historia Natural, 14: 129-138.

Alba-Tercedro, J., Pardo, I., Prat, N. \& Pujante, A. (2005). Protocolos de Muestreo y Análisis para Invertebrados Bentónicos. In Ministerio de Medio Ambiente, Confederación Hidrográfica del Ebro y URS (Eds.), Metodología para el establecimiento del Estado Ecológico según la Directiva Marco del Agua. Madrid, España.

Álvarez-León, R. (1991). Peces colectados en el río Acandí (Chocó), Suroeste del Caribe Colombiano. Caldasia, 16: 525-530. 
Bass, D. (2003). A comparison of freshwater macroinvertebrate communities on small Caribbean islands. Bioscience 53: 1094-1100.

Bell, K. N. I. (2007). Opportunities in stream drift: methods, goby larval types, temporal cycles, in situ mortality estimation, and conservation implications. In N. L. Evenhuis \& J. M. Fitzsimons (Eds.). Bishop Museum Bulletin in Cultural and Environmental Studies 3 (pp. 35-61). Hawaii, USA.

Blanco, J. F. \& Scatena, F.N. (2005). Floods, habitat hydraulics and upstream migration of Neritina virginea (Gastropoda: Neritidae) in Northeastern Puerto Rico. Caribbean Journal of Science, 41: 55-74.

Blanco, J. F. \& Scatena, F. N. (2006). Hierarchical contribution of river-ocean connectivity, water chemistry, hydraulics, and substrate the distribution of diadromous snails in Puerto Rican streams. Journal of the North American Benthological Society, 25: 82-98.

Blanco, J. F. \& Scatena, F. N. (2007). The spatial arrangement of Neritina virginea (Gastropoda: Neritidae) during upstream migration in a split-channel reach. River Research and Applications, 23: 235-245.

Blanco, J. F. (2009a). The hydroclimatology of Gorgona Island: seasonal and ENSO-related patterns. Actualidades Biológicas, 31: 111-121.

Blanco, J. F. (2009b). Características físico-químicas de las quebradas del PNN Gorgona. Actualidades Biológicas, 31: 123-140.

Blanco, J. F., Ramírez, A. \& Scatena, F. N. (2009). The streams of Gorgona Natural Park within the global context: An introduction to the special issue. Actualidades Biológicas, 31: 105-110.

Boulton, A. J., Boyero, L., Covich, A. P., Dobson, M., Lake, S. \& Pearson, R. (2008). Are tropical streams ecologically different from temperate streams?. In D. Dudgeon (Ed.), Tropical stream ecology (pp. 257284.). Elsevier, London, England.

Boyero, L. \& Bailey, R. C. (2001). Organization of macroinvertebrate communities at a hierarchy of spatial scales in a tropical stream. Hydrobiologia, 464: 219-225.

Boyero, L., Ramírez, A., Dudgeon, D. \&. Pearson, R. G. (2009). Are tropical streams really different? Journal of the North American Benthological Society, 28: 397-403.

Cala, P. (1990). Biodiversidad en aguas dulces de la isla. In J. Aguirre \& O. Rangel (Eds.), Biota y ecosistemas de Gorgona (pp. 263-274). Bogotá, Colombia.

Camargo, C. \& Rozo M. P. (2003). Colombian Darien Ephemeroptera. In E. Gaino (Ed.). Research update on Ephemeroptera and Plecoptera (pp. 291-292). University of Perugia. Perugia. Italia.

Carvajal-Quintero, J. D. (2011). El fenómeno de La Viuda: migración de estadios tempranos de peces entre el medio marino y continental en el corregimiento de El Valle, bahía Solano (Chocó-Colombia). (Trabajo de grado). Facultad de Ciencias Exactas y Naturales. Universidad de Antioquia. Medellín, Colombia.

Castellanos-Galindo, G. A., Sanchez, G. C., Beltrán-León, B. S. \& Zapata, L. (2011). A goby-fry fishery in the northern Colombian Pacific Ocean. Cybium, 391: 395.

Cincotta, R., Wisnewski, J. \& Engelman, R. (2000). Human population in the biodiversity hotspots. Nature, 303: 990-992.

Coffman, W. P. \& de la Rosa, C. L. (1998). Taxonomic composition and temporal organization of tropical and temperate species assemblages of lotic Chironomidae. Journal of the Kansas Entomological Society, 71: 388-406.

Contreras, E. (2012). Rasgos biológicos de los insectos acuáticos como respuesta a la perturbación natural por sequía en los ríos y quebradas de la ecorregión Capurganá, Chocó Biogeográfico, Municipio de Acandi. (Trabajo de grado). Facultad de Ciencias Exactas y Naturales. Universidad de Antioquia. Medellín, Colombia.

Craig, D. A. (2003). Geomorphology, development of running water habitats, and evolution of black flies on Polynesian islands. Bioscience, 53: 1079-1093.

Crowl, T. A., McDowell, W. H., Covich, A. P. \& Johnson, S. L. (2001). Freshwater shrimp effects on detrital processing and nutrients in a tropical headwater stream. Ecology, 82: 775-783.

Díaz-Merlano, J. M. \& Gast-Harders, F. (2009). El Chocó biogeográfico de Colombia. Banco de Occidente. Cali, Colombia.

Dudgeon, D. (2000). The ecology of tropical Asian rivers and streams in relation to biodiversity conservation. Annual Review of Ecology, Evolution, and Systematics, 31: 239-263.

Eslava, J. A. (1995). Climatología. In P. Leyva (Ed.). Colombia Pacífico, Tomo I. Obtenido de http://www. banrepcultural.org/blaavirtual/faunayflora/pacific1/ indice.htm).

Fernández, L. \& Schaefer, S. A. (2005). New Trichomycterus (Siluriformes: Trichomycteridae) from an offshore island of Colombia. Copeia, 2005: 68-76.

Fievet, E., Doledec, S. \& Lim, P. (2001). Distribution of migratory fishes and shrimps along multivariate gradients in tropical island streams. Journal of Fish Biology, 59: 390-402.

Flecker, A. S. \& Feifarek, B. P. (1994). Disturbance and the temporal variability of invertebrate assemblages in two Andean streams. Freshwater Biology, 31: 131-142. 
Fowler, H. F. (1944). Fresh-water fishes from Northwestern Colombia. Proceedings of the Academy of Natural Sciences of Philadelphia, 96: 227-248.

Galvis, J. (1980). Un arco de islas terciarias en el occidente colombiano. Geología Colombiana, 11: 7-43.

Galvis, J. \& Mojica, J. (1995). Geología. In P. Leyva (Ed.). Colombia Pacífico, Tomo I. Obtenido de http://www. banrepcultural.org/blaavirtual/faunayflora/pacific1/ indice.htm).

García-Valencia, C. (2007). (Ed). Atlas del golfo de Urabá: una mirada al Caribe de Antioquia y Chocó. Instituto de Investigaciones Marinas y Costeras-Invemar- y Gobernación de Antioquia. Serie de Publicaciones Especiales de Invemar $N^{0} 12$. Santa Marta, Colombia.

García, J., Cantera, J. R., Zuñiga, M. C. \& Montoya, J. (2009). Estructura y diversidad de las comunidades de macroinvertebrados acuáticos en la cuenca baja del río Dagua (Andén Pacífico Vallecaucano-Colombia). Revista de Ciencias-Universidad del Valle, 12: 25-49.

Gómez-Aguirre A. M. (2009). Variación de la estructura y composición de los ensamblajes de macroinvertebrados bénticos en la jerarquía espacial de los sistemas lóticos del Parque Nacional Natural Gorgona. (Trabajo de grado). Facultad de Ciencias Exactas y Naturales. Universidad de Antioquia. Medellín, Colombia.

Gómez-Aguirre, A. M., Longo-Sánchez, M. C. \& Blanco, J. F. (2009). Macroinvertebrate assemblages in Gorgona Island streams: spatial patterns during two contrasting hydrologic periods. Actualidades Biológicas, 31: 161-178.

Gomi, T., Sidle, R. C. \& Richardson, J. S. (2002). Understanding processes and downstream linkages of headwater systems. Bioscience, 52: 905-916.

Greathouse, E. \& Pringle, C. M. (2006). Does the river continuum concept apply on a tropical island? Longitudinal variation in a Puerto Rican stream. Canadian Journal of Fisheries and Aquatic Sciences, 63: 134-152.

Gutierrez-Fonseca P., Ramírez, A., Umaña, G. \& Springer, M. (2013). Macoinvertebrados dulceacuícolas de la Isla del Coco, Costa Rica: especies y comparación con otras islas del Pacífico Tropical Oriental. Revista de Biología Tropical, 61: 657-668.

Jacobsen, D., Cressa, C. \& Dudgeon, D. (2008). Macroinvertebrates: composition, life histories and production. In D. Dudgeon (Ed.), Tropical stream ecology. Elsevier (pp. 65-105). London. England.

Hein, C. L., Pike, A. S., Blanco, J. F., Covich, A. P., Scatena, F. N., Hawkins, C. P. \& Crowl, T. A. (2011). Effects of coupled natural and anthropogenic factors on the community structure of diadromous fish and shrimp species in tropical island streams. Freshwater Biology, 56: 1002-1015.
Hyslop, E. \& Hunte-Brown, M. (2012). Longitudinal variation in the composition of the benthic macroinvertebrate fauna of a typical North coast Jamaican river. Revista de Biología Tropical, 60: 291-303.

IGAC (Instituto Geográfico Agustín Codazzi). (2006). Chocó. Características geográficas. Bogotá. Colombia.

IGAC-INGEOMINAS (Instituto Nacional de Geología y Minas). (2005). Investigación integral del Andén Pacífico colombiano. Bogotá, Colombia.

Keith, P. (2003). Biology and ecology of amphidromous Gobiidae of the Indo-Pacific and the Caribbean regions. Journal of Fish Biology, 63: 831-847.

Kerr, A. C. (2005). La Isla de Gorgona, Colombia: A petrological enigma? Lithos, 84: 77-101.

Kikkert, D. A. \&. Crowl, T. A. (2009). Upstream migration of amphidromous shrimps in the Luquillo Experimental Forest, Puerto Rico: temporal patterns and environmental cues. Journal of the North American Benthological Society, 28: 233-246.

Lobo-Guerrero, A. (1995). Hidrología e hidrogeología. In P. Leyva (Ed.), Colombia Pacífico, Tomo I. Obtenido de http://www.banrepcultural.org/blaavirtual/faunayflora/pacific1/indice.htm).

Lombana, A. (1995). Suelos. In P. Leyva (Ed.), Colombia Pacifico, Tomo I. Obtenido de http://www.banrepcultural.org/blaavirtual/faunayflora/pacific1/indice. htm).

Longo-Sánchez, M. C. \& Blanco, J. F. (2009). Sobre los filtros que determinan la distribución y la abundancia de la fauna diádroma y no diádroma en cada nivel jerárquico del paisaje fluvial en islas. Actualidades Biológicas, 31: 179-195.

Longo-Sánchez, M. \& Blanco, J. F. (2014a). Patterns at Multi-Spatial Scales on Tropical Island Stream Insect Assemblages (Gorgona Island Natural National Park, Colombia, Tropical Eastern Pacific). Revista de Biología Tropical, Vol. 62 (Supp. 1) 65-83.

Longo-Sánchez, M. \& Blanco, J. F. (2014b). Shredders are abundant and species-rich in tropical continentalisland low-order streams (Tropical Eastern Pacific). Revista de Biología Tropical, Vol. 62 (Supp.1) 85-105.

Lorion, C. M., Kennedy, B. P. \& Braatne, J. H. (2011). Altitudinal gradients in stream fish diversity and the prevalence of diadromy in the Sixaola River basin, Costa Rica. Environmental Biology of Fishes, 91: 487-499.

Lyons, J. \& Schneider, D. W. (1990). Factors influencing fish distribution and community structure in a small coastal river in southwestern Costa Rica. Hydrobiologia, 203:1-14.

Macioleck, J. A. \& Ford, J. (1987). Macrofauna and environment of the Nanpil-Kiepw River, Ponape, Eastern 
Caroline Islands. Bulletin of Marine Science, 41: 623-632.

Maldonado-Ocampo, J. A., Ortega-Lara, A., Usma, J. S., Galvis, G., Villa-Navarro, F. A., Vásquez, L., PradaPedreros, S. \& Ardila, C. (2005). Peces de los Andes de Colombia: guía de campo. Instituto Alexander von Humboldt, Bogotá. Colombia.

Martínez, J. O. (1995). Geomorfología. In P. Leyva (Ed.), Colombia Pacífico, Tomo I. Obtenido de http://www. banrepcultural.org/blaavirtual/faunayflora/pacific1/ indice.htm).

McDowall, R. M. (2007). On amphidromy, a distinct form of diadromy in aquatic organisms. Fish and Fisheries, $8: 1-13$.

Mena-Moreno, N. (2010). Estado ecológico de un sistema fluvial tropical costero: Río Capurganá (Darién colombiano). (Trabajo de grado). Corporación Académica Ambiental. Universidad de Antioquia, Turbo, Colombia.

Moncada L. I., Mantilla, J. S., Lotta, I., Matta, N. E. \& Adler, P. (2013). Simulium cormonsi Wygodzinsky (Diptera: Simuliidae) in Colombia: First record, egg and larval descriptions, and bionomics. Proceedings of the Entomological Society of Washington, 115: 103-106.

Myers, N., Mittermeier, R. A., Mittermeier, C. G., da Fonseca G. A. B. \& Kent, J. (2000). Biodiversity hotspots for conservation priorities. Nature, 403: 853-858.

Olson, D. M. \& Dinerstein, E. (2002). The Global 200: Priority ecoregions for global conservation. Annals of the Missouri Botanical Garden, 89: 199-224.

Page, W. D. (1986). Geología sísmica y sismicidad del Noroeste de Colombia. Informe técnico ISA-INTEGRAL-Wood ward \& Clyde Consultants. Medellín, Colombia.

Parsons, M, Thoms, M. C. \& Norris, R. H. (2004). Using hierarchy to select scales of measurement in multiscale studies of stream macroinvertebrate assemblages. Journal of the North American Benthological Society, 23: $157-170$.

Prahl, H, Guhl, F. \& Grogl, M. (1979). Gorgona. Grupo Futura Editorial. Bogotá, Colombia.

Prahl, H. (1983). Hypolobocera gorgonensis sp. nov. (Crustacea: Brachyura: Pseudothelphusidae): un nuevo cangrejo de agua dulce de la isla de Gorgona, Colombia. Cespedesia, 45-46: 105-110.

Prahl, H., Caicedo, C. \& Ríos, R. (1984). Camarones palaemónidos (Crustacea, Caridea, Palaemonidae) de agua dulce y salobre del Valle del Cauca. Cespedesia, 13: 45-57.

Pringle, C. M. (1997). Exploring how disturbance is transmitted upstream: going against the flow. Journal of the North American Benthological Society, 16: 425-438.

Pringle, C. M., Hemphill, N., McDowell, W. H., Bednarek, A. \& March, J. G. (1999). Linking species and ecosystems: different biotic assemblages cause interstream differences in organic matter. Ecology, 80: 1860-1872.

Prüssman, J. (2012). Nuevos elementos para el manejo integrado de la región de Urabá, costa Caribe colombiana. (Tesis de maestría), Universidad EAFIT, Medellín, Colombia.

Ramírez, A. \& Pringle, C. M. (1998a). Invertebrate drift and benthic community dynamics in a lowland Neotropical stream, Costa Rica. Hydrobiologia, 386: 19-26.

Ramírez, A. \& Pringle C. M. (1998b). Structure and production of a benthic insect assemblage in a Neotropical stream. Journal of the North American Benthological Society, 17: 443-463.

Ramírez, A. \& Hernández-Cruz, L. R. (2004). Aquatic insect assemblages in shrimp-dominated tropical streams, Puerto Rico. Biotropica, 36: 259-266.

Ramírez, A. \& Pringle, C. M. (2006). Fast growth and turnover of Chironomid assemblages in response to stream phosphorus level in a tropical lowland landscape. American Society of Limnology and Oceanograph, 5: 189-196.

Ramírez, A., Pringle, C. M. \& Douglas, M. (2006). Temporal and spatial patterns in stream physicochemistry and insect assemblages in tropical lowland streams. Journal of the North American Benthological Society, 25: 108-125.

Resh, V. H. \& De Szalay, E. A. (1995). Streams and rivers of Oceania. In C. E. Cushing, K. W. Cummins \& G. W. Minshall (Eds.), River and stream ecosystems of the World. Elsevier, (pp. 717-736). Amsterdam, Holland.

Sanchez-Garcés, G. C., Castellano-Galindo, G. A., BeltránLeón, B. S. \& Zapata, L. (2011). Algunos aspectos relacionados con la pesca de juveniles de Góbidos diádromos (Perciformes: Gobiidae) en ríos costeros de la vertiente Pacífico de Colombia. En: C. A. Lasso, F. de Paula Gutiérrez, M. A. Morales-Betancourt, E. Agudelo, H. Ramírez-Gil \& R. E. Ajiaco-Martínez (Eds.), II. Pesquerías continentales de Colombia: cuencas del Magdalena- Cauca, Sinú, Canalete, Atrato, Orinoco, Amazonas y vertiente del Pacífico. Serie Editorial Recursos Hidrobiológicos y Pesqueros Continentales de Colombia (pp. 283-289). Bogotá, Colombia.

Scatena, F. N. \& Gupta, A. (2012). Streams of the montane humid tropics. In J. Shroder \& E. Wohl (Eds.), Treatise on geomorphology. Academic. San Diego, USA. 
Schneider, D. W. \& Lyons, J. (1993). Dynamics of upstream migration in two species of tropical freshwater snails. Journal of the North American Benthological Society, 12: 3-16.

Smith, G. C., Covich, A. P. \& Brasher, A. M. D. (2003). An ecological perspective on the biodiversity of tropical island streams. Bioscience, 53: 1048-1051.

Turner, D., Williams, D. D. \& Alkins-Koo, M. (2008). Longitudinal changes in benthic community composition in four Neotropical streams. Caribbean Journal of Science, 44: 380-394.

UAESPNN-DTNPO. (2006). Parque nacional natural Utría plan de manejo 2005-2009. Bahía Solano Colombia. Unidad Administrativa Especial del Sistema de Parques Nacionales Naturales de Colombia. Medellín, Colombia.
UAESPNN-DTSO. (2005). Plan de Manejo Parque Nacional Natural Gorgona. Resumen Ejecutivo. SUT 021105. Unidad Administrativa Especial del Sistema de Parques Nacionales Naturales de Colombia-Dirección Territorial Suroccidental. Cali, Colombia.

Valencia, S. M., Pérez, G. A., Lizarazo, P. X. \& Blanco, J. F. (2009). Patrones espacio-temporales de la estructura y composición de la hojarasca en las quebradas del Parque Nacional Natural Gorgona. Actualidades Biológicas, 31: 197-211.

Vannote, R., Minshall, G., Cummins, K., Sedell, J. \& Cushing C. (1980). The river continuum concept. Canadian Journal of Fisheries and Aquatic Sciences, 37: 130- 137.

Winterbourn, M. J., Rounick, J. S. \& Cowie, B. (1981). Are New Zealand stream ecosystems really different? New Zealand Journal of Marine \& Freshwater Research, 15: 321-328. 